\title{
A Machine Learning Approach For Simulating Ground Penetrating Radar
}

\author{
Iraklis Giannakis, Antonios Giannopoulos \\ Institute for Infrastructure and Environment \\ School of Engineering \\ The University of Edinburgh \\ Edinburgh, UK \\ Email: i.giannakis@ed.ac.uk, a.giannopoulos@ed.ac.uk
}

\author{
Craig Warren \\ Future Engineering Department \\ Mechanical and Construction Engineering \\ Northumbria, UK. \\ Email: craig.warren@ northumbria.ac.uk
}

\begin{abstract}
The ability to produce, store and analyse large amounts of well-labeled data as well as recent advancements on supervised training, led machine learning to gain a renewed popularity. In the present paper, the applicability of machine learning to simulate ground penetrating radar (GPR) for high frequency applications is examined. A well-labelled and equally distributed training set is generated synthetically using the finitedifference time-domain (FDTD) method. Special care was taken in order to model the antennas and the soils with sufficient accuracy. Through a stochastic parameterisation, each model is expressed using only seven parameters (i.e. the fractal dimension of water fraction, the heigh of the antenna and so on). Based on these parameters and the synthetically generated training set, a machine learning framework is trained to predict the resulting A-Scan in real-time. Thus, overcoming the time-consuming calculations required for an equivalent FDTD simulation.
\end{abstract}

Keywords-FDTD, GPR, machine learning, neural networks.

\section{INTRODUCTION}

Numerical modelling can shed a light on scattering mechanisms and furthermore give us an insight on GPR's performance. The obvious requirement for this to happen is that the model is an adequate approximation of reality. Numerical modelling has been extensively used for qualitative studies using simplified sensors and generic targets buried in halfspaces [1], [2], [3]. Some efforts have also been made for simulating cluttering sources [4] within the ground as well as incorporating an accurate representation of the antenna system [5], [6], [7]. In [8], [9] a numerical framework is suggested that tries to realistically simulate GPR for AP landmine detection incorporating soil's inhomogeneities, rough surface, dispersive soils, vegetation, realistic targets and accurate representations of two GPR commercial systems [14]. The numerical framework was applied using gprMax [10], an open software that solves Maxwell's equations using a second-order accuracy finite-difference time-domain (FDTD) method [11], [12].

The numerical framework suggested in [8], [9] has the potential to evaluate GPR's performance for a given scenario. Nonetheless, FDTD is a time consuming algorithm with large computational requirements that still are not trivially available to everyone. To overcome this obstacle, a machine learning scheme was suggested in [13] which tries to predict the signal to clutter ratio (SCR) based on a given set of parameters. The regression model was trained based on an equally distributed set of synthetic data. The latter were calculated using the numerical framework suggested in [8], [9] applied in a 2DFDTD solver (due to computational constrains [13]). The posttraining outcome of this approach is a two-layered neural network that predicts the SCR based on a set of given parameters (sand, fraction, fractal dimension of soil's surface, depth of the target and so on). Although the training process is computationally expensive, nonetheless, once trained, the resulting scheme can be applied in real time using minimum computational requirements.

In the present paper, following the notion of [13], a machine learning framework is suggested that is trained to predict the full GPR's waveform in time domain. In contrast to [13], that predicts SCR (scalar), the present technique has the ability to calculate (in real time) the full GPR's response (vector). In addition, the suggested regression model is trained using fully $3 \mathrm{D}$ models incorporating accurate representations of a commercial antenna system [10].

The proposed methodology overcomes the large computational resources necessary for a 3D-FDTD simulation. The resulting regression model acts as a real-time numerical simulator that uses model's parameters as inputs (water fraction of soil, heigh of the antenna and so on) and gives as output (in real time) the full response of GPR for the given inputs.

\section{Generating Training Set}

\section{A. Parameterisation of the model}

In the present paper a regression model based on neuralnetworks is presented that is used to predict in real time the response of GPR given a set of parameters. The necessary parameters to describe the model are the following:

- $\quad$ sand fraction $(S)$

- minimum volumetric fraction $(m)$

- maximum volumetric fraction $(M)$

- $\quad$ spatial statistics of water volumetric fraction $\left(\beta_{w}\right)$

- maximum absolute deviation of the surface $(T)$

- $\quad$ spatial statistics of soil's surface (roughness) $\left(\beta_{T}\right)$

- heigh of the antenna $(H)$

The antenna used in the present paper is a model representation of $1.5 \mathrm{GHz}$ GSSI as described in [15]. By selecting 


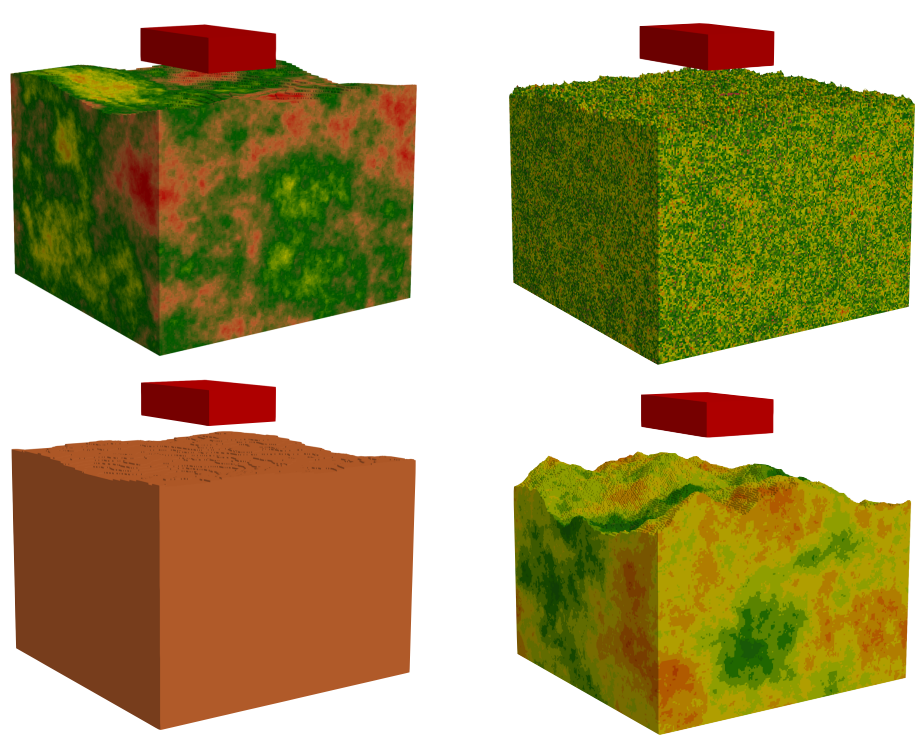

Fig. 1. Four randomly selected models from the set used to train the regression scheme.

the parameters above in a stochastic manner following the approach described in [13] an equally distributed and adequate training set is constructed. The size of model is $40 \times 40 \times 50$ $\mathrm{cm}$ and the discretisation step is $1 \mathrm{~mm}$. The time step is chosen based on the Courant stability conditions [12].

Recent edition of gprMax [10] has the ability to realise the above models in a straightforward manner. Thus, gprMax is selected to synthetically generate the training set. In addition, gprMax allows the usage of graphics processing units (GPU) making the procedure computationally affordable. A set consisted of two thousand stochastically selected models (see Fig. 1) is proven adequate for the current application. Although generating the training set and training the regression scheme is time consuming, nonetheless, the post-trained regression scheme works in real-time.

\section{B. Reducing the dimensionality of the problem}

The trace is consisted of 3000 points and any regression scheme should be capable of predicting a relatively large vector using seven input parameters. This increases the complexity of the problem and create instabilities during the training process that result to noisy outputs. This can be partially solved by under-sampling traces every ten time steps (i.e. removing high frequencies with null content). To reduce the dimensions of the data furthermore, principal component analysis (PCA) is employed. PCA maps the data to its principal orthogonal axes from which the data can be reconstructed through a linear combination of those. The principal axes of the training set consists of 300 traces and its trace has 300 points. A linear combination of these 300 traces can be reproduce every trace of the training set. Figure (2) illustrates the 5 most dominant axes.

To reduce the dimensionality of the training set, the 45 most dominant axes are chosen and the best linear combination of those that minimise the squared error between the actual traces
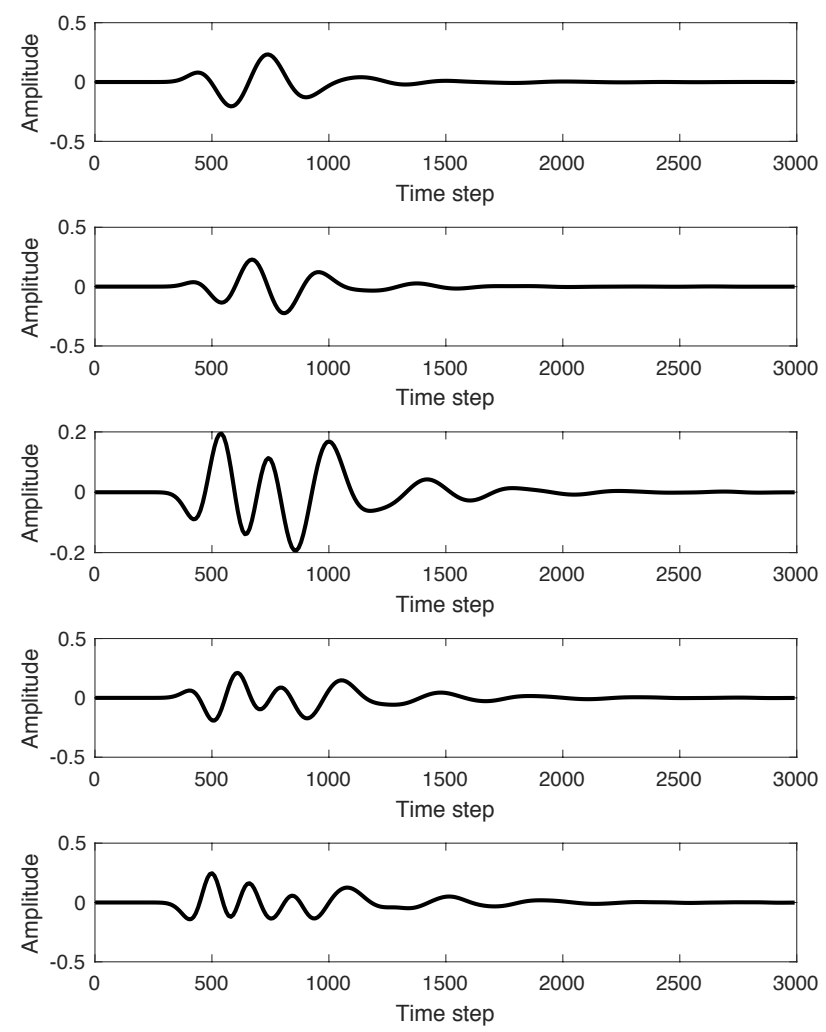

Fig. 2. The 5 dominant axes of the training set as calculated using PCA.

and their compressed edition is evaluated

$$
\mathbf{Y}=\mathbf{A} \mathbf{w}
$$

where $\mathbf{Y}$ is a vector that represents the actual trace, $\mathbf{A}$ is a matrix that contain the 45 dominant axes of the training set and $\mathbf{w}$ is a vector containing the 45 linear coefficients. The vector $\mathbf{w}$ can be calculated in a straightforward manner using the least-squares method $\mathbf{w}=\left(\mathbf{A}^{T} \mathbf{A}\right)^{-1} \mathbf{A}^{T} \mathbf{Y}$. Thus, all the traces can be reproduced using a shared matrix $\mathbf{A}$ and their unique linear coefficients $\mathbf{w}$. The latter has 45 dimensions in contrast to 3000 dimensions that the initial data had. Obviously some information is lost during this compression, nonetheless using 45 principal axes has negligible affects as illustrated in Fig. 3.

\section{REGRESSION MODEL}

Using the input parameters (sand fraction, fractal dimension of surface etc.) different models are generated having the same statistical properties i.e. the same fractal dimension of water fraction and the same fractal dimension of topography. Each model will give rise to different A-Scans all resulted from models with the same statistical properties. The algebraic relationship between the input parameters and their resulting average trace is evaluated here using neural networks (NN).

The NN architecture is trained using the training set described previously. The set is divided into training (80\%), validation (10\%) and testing set $(10 \%)$. The validation set is used in each iteration of the training to check if the NN overfit. The testing set is used to evaluate the performance of the NN 


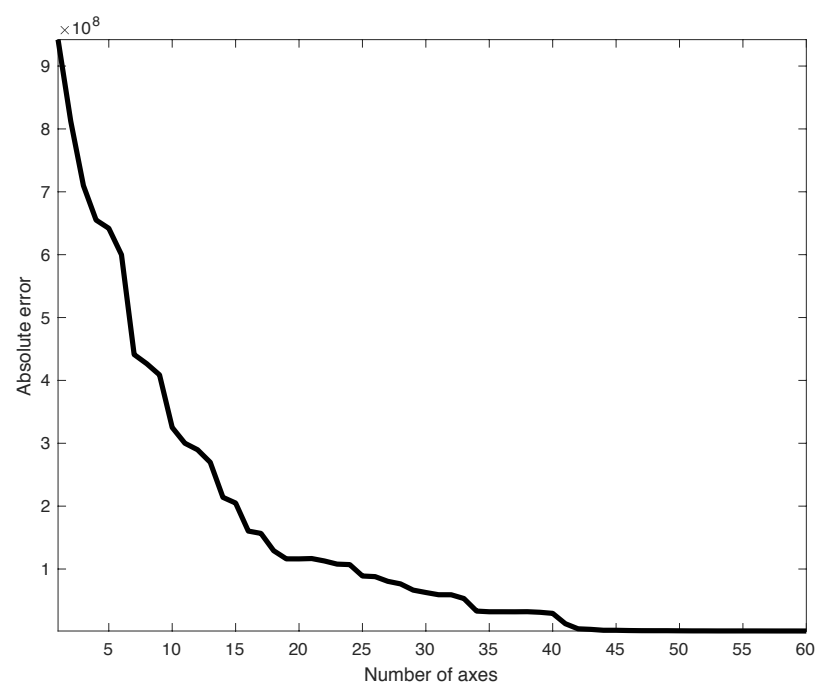

Fig. 3. Absolute error of the training set with respect to the number of axes used to approximate it.

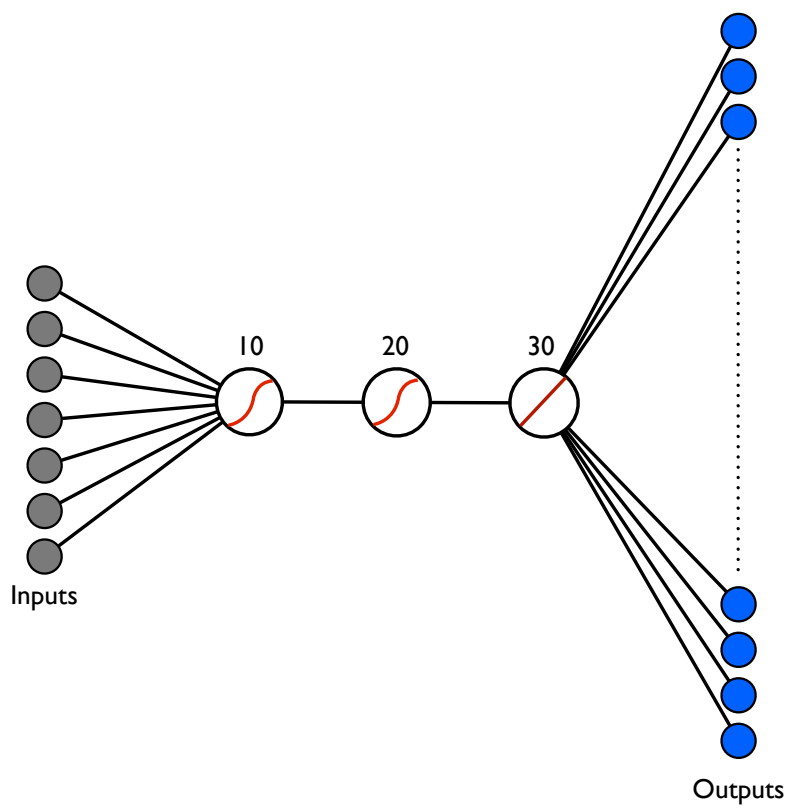

Fig. 4. The neural network architecture consists of three layers of 10 , 20 and 30 neutrons respectively. The first two layers use sigmoid activation functions while the third layer uses a linear one. The inputs parameters are the parameters that define the model (sand fraction, water volumetric fraction etc.) and the output is the compressed A-Scan.

in unknown data and lastly, the training set is used to fine-tune the NN.

The NN architecture is chosen through a trial and error process and it is illustrated in Fig. 4. A three-hidden layer NN is used with 10, 20 and 30 neutrons respectively. The first two layers use sigmoid activation functions while the last layer uses a linear one in order to predict the continuous nature of the compressed A-Scan. A complex-conjugate gradient method is used during the training.

Fig. 5 illustrates six randomly selected cases used to validate the proposed $\mathrm{NN}$ architecture. The average traces
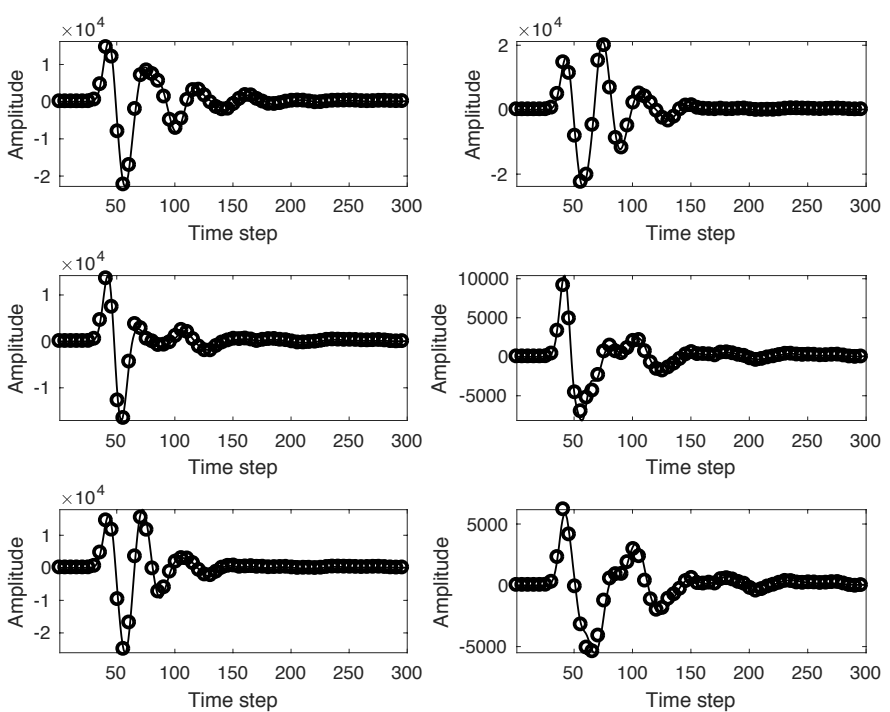

Fig. 5. Six randomly selected scenarios chosen to illustrate the validity of the trained NN. With solid line is the average trace calculated using FDTD. With dotted lines are the predicted A-Scans (in real time) using the suggested regression scheme.

evaluated using FDTD are compared with the predicted compressed traces using NN. Fig. 5 highlights the accuracy of the $\mathrm{NN}$ at predicting the average trace for given input parameters. Notice that NN evaluate A-Scan in real time in contrast to FDTD that (depending on computational resources available) is considered a computational expensive and time consuming technique.

\section{CONCLUSIONS}

A novel method to simulate GPR, alternative to traditional techniques like FDTD is presented. It is based on a NN regression scheme which is trained using numerically evaluated data. The inputs of the NN are model parameters that uniquely define a case study (sand fraction, water fraction, antenna heigh etc.). The output is the predicted trace. PCA is used in order to reduce the size of the A-Scans in order to avoid complexities arising in training $\mathrm{NN}$ with high dimensional outputs. The suggested scheme was validated at unknown cases that were not included during the training process. The traces evaluated using FDTD and the ones predicted using NN are in good agreement indicating the validity of the proposed framework. The fact that NN can run in real time can vastly decrease the computational cost of FDTD, thus used as an accurate and efficient forward solver for specific parameterised environments.

\section{REFERENCES}

[1] L. Gurel and U. Oguz, "Three-dimensional FDTD modelling of a groundpenetrating radar," IEEE Trans. Geosci. Remote Sens., vol. 38, no. 4, pp. 1513-1521, Jul. 2000.

[2] U. Oguz and L. Gurel, "Frequency responses of ground-penetrating radars operating over highly lossy grounds," IEEE Trans. Geosci. Remote Sens., vol. 40, no. 6, pp. 1385-1394, Jun. 2002/

[3] L. Gurel and U. Oguz, "Optimization of the transmitter?receiver separation in the ground-penetrating radar," IEEE Trans. Geosci. Remote Sens., vol. 51, no. 3, pp. 362-370, Mar. 2003. 
[4] L. Gurel and U. Oguz, "Simulations of ground-penetrating radars over lossy and heterogeneous grounds,? IEEE Trans. Geosci. Remote Sens., vol. 39, no. 6, pp. 1190-1197, Jun. 2001.

[5] M. A. Gonzalez-Huici, U. Uschkerat and A. Hoerdt, "Numerical simulation of electromagnetic-wave propagation for land mine detection using GPR," in Proc. IEEE Inter. Geosci. Remote Sens, pp. 4957-4960, 23-28 Jul. 2007.

[6] M. A. Gonzalez-Huici and U. Uschkerat, "GPR modeling for landmine detection," in Proc. Int. Symp. Electromagn. Theory, pp. 152-155, Aug. 2010.

[7] M. A. Gonzalez-Huici, "A strategy for landmine detection and recognition using simulated GPR responses," in Proc. Int. Conf. Ground Penetrat. Radar (GPR), Brussels, Belgium, pp. 871-876, Jun. 2012.

[8] I. Giannakis, A. Giannopoulos and C. Warren, "A realistic FDTD numerical modeling framework of ground penetrating radar for landmine detection," IEEE Journ. of Sel. Top. in App. Earth Obser. and Rem. Sens., vol. 9, no. 1, pp. 37-51, Jan. 2016.

[9] I. Giannakis, A. Giannopoulos, and N. Davidson, "Realistic modelling of ground penetrating radar for landmine detection using FDTD," in Proc. Int. Conf. GPR, Brussels, Belgium, 2014, pp. 983-989.

[10] C. Warren, A. Giannopoulos and I. Giannakis, "gprMax: Open source software to simulate electromagnetic wave propagation for Ground Penetrating Radar," Comp. Phys. Communication, vol. 209, pp. 63-170, 2016.

[11] S. Yee, "Numerical solution of initial boundary value problems involving Maxwell's equations in isotropic media," IEEE Trans. Antennas Propag., vol. 14, no. 3, pp. 302-307, May 1966.

[12] A. Taflove and S. C. Hagness, Computational Electrodynamics, the Finite-Difference Time-Domain Method, 2nd ed. Norwood, MA, USA: Artech House, 2000.

[13] I. Giannakis, A. Giannopoulos, and A. Yarovoy, "Model-based evaluation of signal-to-clutter ratio for landmine detection using groundpenetrating radar," IEEE Trans. Geosci. Remote Sens., vol. 54, no. 6, pp. 3564-3573, 2016.

[14] C. Warren and A. Giannopoulos, "Creating finite-difference timedomain models of commercial ground-penetrating radar antennas using Taguchi's optimisation method," Geophysics, vol. 76, no. 2, pp. G37G47, Apr. 2011.

[15] I. Giannakis, A. Giannopoulos, and C. Warren, "A hybrid linear/nonlinear full waveform inversion for modelling commercial GPR antennas using FDTD," IEEE Trans. Geosci. Remote Sens., submitted. 